\begin{tabular}{|c|c|c|c|}
\hline \multirow[t]{2}{*}{ Studien } & Erhaltungstherapie & PFS & Gesamtüberleben \\
\hline & & HR (95\%-KI) & HR (95\%-KI) \\
\hline CAIRO3 & $\begin{array}{l}\text { Capecitabin/ } \\
\text { Bevacizumab }\end{array}$ & $0,43(0,36-0,51)$ & $0,89(0,73-1,09)$ \\
\hline AIO-KRK-0207 & $\begin{array}{l}\text { Fluoropyrimidin/ } \\
\text { Bevacizumab }\end{array}$ & $0,49(0,38-0,63)$ & $0,99(0,71-1,38)$ \\
\hline AIO-KRK-0207 & Bevacizumab & $0,64(0,50-0,82)$ & $0,88(0,63-1,23)$ \\
\hline SAKK-41/06 & Bevacizumab & $0,75(0,58-0,97)$ & $0,83(0,61-1,13)$ \\
\hline Gesamt & & $0,56(0,43-0,72)$ & $0,89(0,78-1,02)$ \\
\hline
\end{tabular}

freiheit) von großer Bedeutung, und die Daten implizieren, dass die Erkrankung im weiteren Verlauf onkologisch so kontrolliert werden kann, dass kein Überlebensnachteil entsteht. Die Daten der CAIRO3-Studie legen dagegen nahe, dass bei kürzerer Induktionschemotherapie von nur 4,5 Monaten eine Erhaltungstherapie im Trend zu einem Überlebensvorteil bei Patienten mit gutem Ansprechen führt (18,8 vs. 24,1 Monate) oder bei Patienten mit synchronen Metastasen und reseziertem Primarius (18 vs. 25 Monate). Letzteres wurde in der AIO-KRK-0207-Studie aber nicht beobachtet.

Bei Patienten mit RAS-mutiertem Tumor muss sicherlich aufgrund der aggressiveren Tumorbiologie im Falle der Entscheidung für eine Therapiepause ein besonders engmaschiges Monitoring erfolgen. Bei Patienten, die auf die Induktionschemotherapie nur mit SD reagiert haben, ist aufgrund der mäßig ausgeprägten Chemosensibilität des Tumors eine Erhaltungstherapie sinnvoll. Hier ist ggf. frühzeitig eine aktive Zweitlinientherapie einzuleiten.

Letztlich gilt es anzumerken, dass sich die Frage einer Bevacizumab-haltigen Erhaltungstherapie in der Erstlinientherapie in der Regel nur bei Patienten mit einem RASmutierten Tumor stellt. Denn RAS-WildtypTumoren sollten in der Erstlinie präferenziell mit Chemotherapie plus einem Antikörper gegen den Rezeptor des epithelialen Wachstumsfaktors (EGFR) behandelt wer- den (AIO-KRK-Leitgruppenempfehlung). Für diese Anti-EGFR-haltige Erstlinientherapiesituation liegen noch keine belastbaren Daten hinsichtlich einer Maintenance/ Deeskalation vor. Da der EGFR-Antikörper in den meisten Phase-III-Studien (Crystal, PRIME, FIRE-3) aber nur ca. 6 Monate appliziert wurde, besteht hier die Möglichkeit einer Deeskalation, ohne dass das in diesen Studien beobachtete, lange OS kompromittiert wird. Entsprechende Studien (AIOKRK-0212, PANAMA) laufen aktuell. Nach einer Induktionschemotherapie werden aktive Therapiepausen zunehmen, insbesondere bei steigendem Einsatz von Lokaltherapieverfahren (RadiofrequenzThermoablation [RFTA], intensitätsmodulierte Strahlentherapie [IMRT], Resektion) in der oligometastasierten Situation im Sinne eines palliativen Debulkings einzelner problematischer Metastasenlokalisationen (Daten der CLOCC-Studie [Ruers T et al. J Clin Oncol. 2015; 33(Suppl):Abstr 3501]).

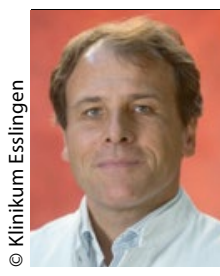

Prof. Dr. med. Michael Geißler Klinik für Allgemeine Innere Medizin, Onkologie / Hämatologie, Gastroenterologie und Infektiologie, Klinikum Esslingen gastro@klinikumesslingen.de

\title{
Metastasen raus beim Kolorektalkarzinom
}

Treten Metastasen eines kolorektalen Karzinoms (CRC) in einem anderen Organ auf, ist klar: Sie sollten raus. Nicht so klar war bisher das Vorgehen, wenn zeitgleich oder in kurzem Abstand gleich mehrere Organe befallen werden.

W erden Metastasen eines CRC in Lunge und/oder Leber möglichst vollständig reseziert, überleben immerhin $40 \%$ der Patienten 5 Jahre. Treten Lungen- und Lebermetastasen gemeinsam auf, ist das Management allerdings nicht eindeutig vorgegeben.

Eine internationale Arbeitsgruppe analysierte das prospektive LiverMetSurvey-Register mit Blick auf simultan auftretende Lungen- und Lebermetastasen (SLLM). Patienten, die in den Jahren 2000 bis 2012 in kurativer Absicht ope- riert worden waren, wurden in 3 Gruppen eingeteilt:

-Gruppe 1 (Kontrolle): Patienten mit isolierten Lebermetastasen $(n=9.185)$,

-Gruppe 2: SLLM-Patienten mit resezierten Leber- und Lungenmetastasen $(\mathrm{n}=149)$,

-Gruppe 3: SLLM-Patienten mit resezierten Lebermetastasen und unresezierten oder nicht resezierbaren Lungenmetastasen $(\mathrm{n}=285)$.

Das adjustierte Überleben wurde anhand von 10 Variablen ausgewertet (Al- ter, Zahl der Lebermetastasen, Synchronizität von Lebermetastasen mit Primarius, CEA-Spiegel, Lymphknoten-Status, initiale Resezierbarkeit der Lebermetastasen sowie Inklusion in Gruppe 3). Die 5-Jahres-Überlebensraten in Gruppe 1 und 2 waren ähnlich (51,5 bzw. 44,5\%), in Gruppe 3 mit 14,3\% deutlich schlechter $(p=0,001)$. Diese Patienten lebten im Vergleich zur Gruppe 1 am kürzesten mit einer Hazard Ratio von 1,77.

Fazit: Bei simultan aufgetretenen Leberund Lungenmetastasen sollte jede Möglichkeit zu einer Operation genutzt werden.

Christina Berndt

Andres A et al. Surgical management of patients with colorectal cancer and simultaneous liver and lung metastases. Br J Surg. 2015;102(6):691-9. 